\title{
Inhibition of v-rel-Induced Oncogenesis through microRNA Targeting
}

\author{
Yongxiu Yao ${ }^{1, *}$, Yaoyao Zhang ${ }^{1}$, Na Tang ${ }^{1,2}$, Miriam Pedrera ${ }^{1}$, Zhiqiang Shen ${ }^{2}$ \\ and Venugopal Nair ${ }^{1, *}$ \\ 1 The Pirbright Institute \& UK-China Centre of Excellence for Research on Avian Diseases, Pirbright, \\ Ash Road, Guildford, Surrey GU24 0NF, UK; zhangyaoyao3848@126.com (Y.Z.); \\ na.tang@pirbright.ac.uk (N.T.); miriam.pedrera@pirbright.ac.uk (M.P.) \\ 2 Binzhou Animal Science and Veterinary Medicine Academy \& UK-China Centre of Excellence for Research \\ on Avian Diseases, Binzhou 256600, Shandong, China; bzshenzq@163.com \\ * Correspondence: yongxiu.yao@pirbright.ac.uk (Y.Y.); venugopal.nair@pirbright.ac.uk (V.N.); \\ Tel.: +44-(0)1483-231-493 (Y.Y.); +44-(0)1483-231-415 (V.N.)
}

Received: 27 March 2018; Accepted: 3 May 2018; Published: 5 May 2018

\begin{abstract}
Several studies have shown that microRNA-targeting is an effective strategy for the selective control of tissue-tropism and pathogenesis of both DNA and RNA viruses. However, the exploitation of microRNA-targeting for the inhibition of transformation by oncogenic viruses has not been studied. The v-rel oncoprotein encoded by reticuloendotheliosis virus $\mathrm{T}$ strain (Rev-T) is a member of the rel/NF- $\mathrm{kB}$ family of transcription factors capable of transforming primary chicken spleen and bone marrow cells. Here, by engineering the target sequence of endogenous microRNA miR-142 downstream of the v-rel gene in a Replication-Competent ALV (avian leukosis virus) long terminal repeat (LTR) with a splice acceptor (RCAS) vector and using a v-rel-induced transformation model of chicken embryonic splenocyte cultures, we show that hematopoietic-specific miR-142 can inhibit the v-rel-induced transformation, and that this inhibition effect is due to the silencing of v-rel expression. The data supports the idea that microRNA-targeting can be used to inhibit viral oncogene-induced oncogenesis.
\end{abstract}

Keywords: microRNA-targeting; inhibition; v-rel-induced transformation; hematopoietic specific miRNA

Avian retroviruses are a group of major pathogens associated with different types of cancers in poultry. Historical studies led by a number of eminent scientists—including Jan Svoboda, whom we are recognizing in this Special Issue-have provided important insights into viruses and cancer [1,2]. Pathogenic avian retroviruses include the different subgroups of avian leukosis viruses (ALV) and their acutely transforming variants such as MC29 and MH2, both of which encode the oncogene v-myc [3].

While protein-coding genes such as oncogenes have played a major role in our understanding of viral pathogenesis over the last several decades, non-coding RNAs such as microRNAs (miRNAs) have also recently emerged as major regulators of complex biological processes and diseases [4]. As many miRNAs are expressed in a distinctive cell type-specific manner [5-7], artificial miRNA target sites have been utilized to regulate gene expression. Vectors expressing miRNA target sites which are designed to act as a decoy or sponge for the cognate miRNA, thus interfering with the ability of the miRNA to regulate its natural targets, have been used to study the role of miRNAs in cancer, cardiac function, and hematopoiesis [8-11]. The miRNA targeting of transgene or viral genome by incorporating tissue-specific miRNA target sequences has provided an effective new strategy to improve the specificity and efficacy of gene and stem cell therapy, to reduce the toxicity of oncolytic viruses, and to attenuate viruses to be used in vaccine development [12-17]. Initially tested 
for picornaviruses $[14,18]$, the cell-specific miRNA-dependent suppression of virus replication has proved to be effective in vivo for both DNA and RNA viruses [13,16,19-22].

The avian retrovirus reticuloendotheliosis virus strain $\mathrm{T}$ (Rev- $\mathrm{T}$ ) encodes the v-rel oncoprotein, which is a member of the Rel/NF-kB transcription factor family. v-rel provides a valuable model for studying NF- $\mathrm{kB}$ signaling in lymphoid cell cancers because of its ability to transform chicken hematopoeitic cells $(12,15)$. Previously, we developed a v-rel-mediated in vitro transformation model of embryonic splenocytes using a Replication-Competent ALV (avian leukosis virus) long terminal repeat (LTR) with a splice acceptor (RCAS) vector [23] that expresses v-rel [24]. This represents a very rapid and efficient method to transform chicken splenocytes into cancer cells and immortalized cell lines.

Despite the fact that miRNA targeting has been widely exploited to attenuate or control virus replication, the inhibition effect of miRNA targeting on virus-induced oncogenesis has not yet been studied. miR-142 was first described as a hematopoietic-specific miRNA in mice [25]. Studies have elucidated multiple important regulatory roles of miR-142 in many biological processes and associated signaling pathways during embryonic development, homeostasis, and disease [26]. miR-142 targeting has been used to prevent retrovirally-encoded transgene expression in antigen-presenting cells while allowing long-term transgene expression in other cells [17], as well as to interrupt the picornavirus lifecycle [20]. In the present study, we sought to determine whether the miRNA targeting approach can be used to inhibit oncogenic virus-induced oncogenesis using Rev-T encoded $\mathrm{v}$-rel-induced transformation as a model. We first explored the silencing of v-rel expression in DF-1 cells by co-infection of RCAS(B) virus expressing miR-142 and RCAS(A) virus expressing v-rel with the engineered miR-142 target sequence. We next investigated the inhibition of $\mathrm{v}$-rel-induced transformation by endogenous miR-142 in embryonic splenocytes. To determine if $\mathrm{v}$-rel-induced transformation can be inhibited by the miRNA targeting approach, we inserted four tandem target sequences of miR-142-3p downstream of the v-rel fused with GFP (green fluorescent protein) in RCAS vector RCAS(A)-v-rel-GFP by introducing annealed oligos miR-142T-F and miR-142T-R (Table 1) into NotI and SpeI sites, generating RCAS-A-v-rel-GFP-142T (Figure 1a). A negative control miRNA [27] target sequence was cloned in the same way using primers miR-NST-F and miR-NST-R (Table 1), generating RCAS-A-v-rel-GFP-NST (Figure 1a).

a

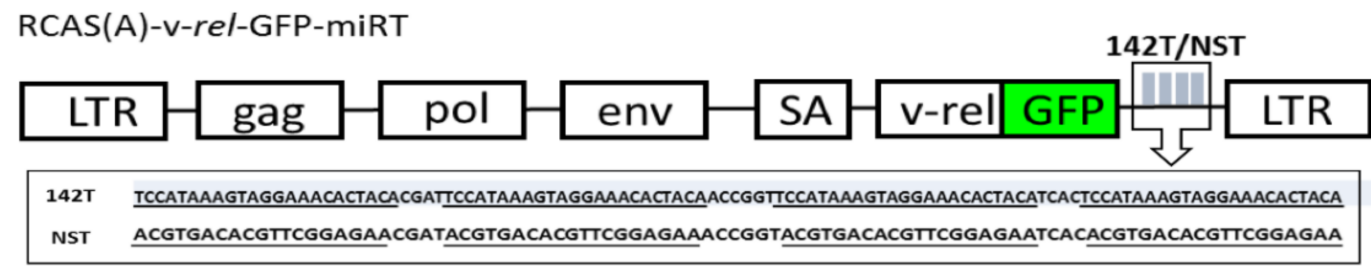

b

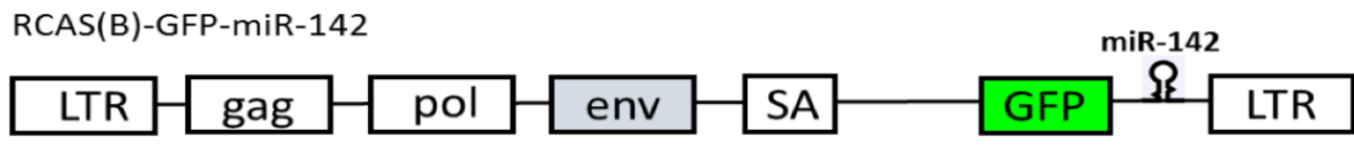

Figure 1. Schematic diagrams of Replication-Competent ALV (avian leukosis virus) LTR with a splice acceptor (RCAS) viruses. LTR, long terminal repeats; GFP, green fluorescent protein; SA, splice acceptor. (a) RCAS(A)-v-rel-GFP-miRT. Four tandem copies of miR-142-3p or non-silencing miRNA target sequence with the spacer sequences were inserted downstream of GFP; (b) RCAS(B)-GFP-miR-142. Pre-miR-142 sequence was inserted downstream of GFP. 
Table 1. List of primers used for the insertion of the miRNA target sequence, miR-142 cloning, and RCAS virus replication detection.

\begin{tabular}{ll}
\hline Primer & Sequence $\mathbf{( 5}^{\prime} \mathbf{-} \mathbf{3}^{\prime} \mathbf{)}$ \\
\hline miR-142T-F & GGCCGCTCCATAAAGTAGGAAACACTACACGATTCCATAAAGTAGGAAACACTACAACCGGTT \\
& CCATAAAGTAGGAAACACTACATCACTCCATAAAGTAGGAAACACTACAA \\
miR-142T-R & GGCCGCACGTGACACGTTCGGAGAACGATACGTGACAC \\
& CACGTGACACGTTCGGAGAAACACGTTCGGAGAATCA \\
& GGCCGCACGTGACACGTTCGGAGAACGATACGTGACAC \\
miR-NST-F & GTTCGGAGAAACCGGTACGTGACACGTTCGGAGAATCA \\
& CACGTGACACGTTCGGAGAAA \\
miR-NST-R & CTAGTTCTCCGAACGTGTCACGTGTGATTCTCCGAACG \\
miR-142-F & TGTCACGTACCGGTTCTCCGAACGTGTCACGTATCGTTCTCCGAACGTGTCACGTGC \\
miR-142-R & GGCCATAATGGCCAGGCGGCCAGCACAGAACTCCTAC \\
GFP-F & ATGGTGAGCAAGGGCGA \\
GFP-R & CCGGTGGTGCAGATGAAC \\
v-rel-F & ATGGACTTTCTCACCAACCTCCG \\
v-rel-R & CGAACGATACCCGACTTG \\
HA & GGATGAGGTGACTAAGAAAG \\
envA & AGAGAAAGAGGGGCGTCTAAGGAGA \\
\hline
\end{tabular}

The vector contains the GFP marker which allows the visualization of infected cells. $\mathrm{v}$-rel expression is expected to be downregulated in the presence of miR-142. First, we examined the inhibition effect of v-rel expression in DF-1 cells. Due to the lack of miR-142 expression in DF-1 cells, we co-infected RCAS(A)-v-rel-GFP-miRT (142T or NST) virus together with an miR-142 expression RCAS virus, RCAS(B)-GFP-miR-142 (Figure 1b). The pre-miR-142 sequence was amplified using primers miR-142-F and miR-142-R and inserted downstream of GFP in RCAS(B)-GFP. The expression of v-rel and miR-142 by the corresponding virus was confirmed by Western blotting and miRNA qRT-PCR (Taqman), respectively. As shown in Figure 2, v-rel is highly expressed when v-rel-142T/v-rel-NST is used in infections, whereas v-rel expression is greatly decreased when the miR-142-expressing virus is co-infected with v-rel-142T but not with v-rel-NST (Figure 2b). As a negative control, no v-rel is expressed in cells infected with miR-142-expressing virus. The expression of miR-142 is confirmed by miRNA Taqman on RNA extracted from the same samples (Figure 2a). miR-155 is not expressed in DF-1. Consistent with the previous finding that $\mathrm{v}$-rel [24] induces miR-155 expression, miR-155 is highly expressed in DF-1 cells when v-rel is expressed (Figure 2c). The miR-155 level measured by miRNA Taqman in infected DF-1 does correlate with the v-rel level, as miR-155 expression is significantly downregulated when v-rel is silenced by miRNA targeting (Figure 2c).

Having demonstrated the inhibition of v-rel expression through miRNA targeting in DF-1 cells in the presence of miR-142, we next examined the potential inhibition of v-rel-induced transformation in embryonic splenocytes. As the mechanisms of v-rel-induced transformation have been extensively studied [28-30] and the v-rel-mediated in vitro transformation model of embryonic splenocytes has been established in our laboratory [24], the transformation properties of the cells are not investigated in this study. As described previously [24], we infected embryonic splenocytes isolated from 19-day old embryos with RCAS(A)-v-rel-GFP, RCAS(A)-v-rel-GFP-142T, or RCAS(A)-v-rel-GFP-NST and harvested cells at day 0 (before infection) and days 2, 6, and 14 post-infection for cell counting, Western blotting, and RNA extraction. The uninfected cells were used as a control. As shown in Figure 3a, the cell number increased initially at day 2 and decreased at day 6 post-infection for both infected and uninfected cells. After six days post-infection, uninfected cells and untransformed cells gradually died off and no cells were left at 14 days post-infection, whereas the transformed cells continued proliferating for both RCAS(A)-v-rel-GFP and RCAS(A)-v-rel-GFP-NST infection groups. The expression of v-rel-GFP protein was assessed by Western blotting, using HY87 antibody [31] for v-rel expression. As shown in Figure 3b, only the transformed cells at the last time point showed an expected band, whereas the level of v-rel expression at other time points was too low to be detected. $\alpha$-tubulin was used for the 
loading control. Due to the undetectable level of v-rel expression before transformation, we measured the v-rel transcript level by RT-PCR with a random primer for reverse transcription and v-rel specific primers v-rel-F and v-rel-R for PCR (Figure 3c). Both GFP (GFP-F and GFP-R) and viral specific primers (HA and envA) for ALV-A virus [32] were used as controls. The primer sequences are listed in Table 1. Although the perfectly complementary miR-142-3p targets were inserted, there is no difference at the transcript level between the transformed and untransformed groups, indicating no direct catalytic cleavage of the viral RNA genome and mRNA. However, the result does reflect a similar level of RCAS virus replication between the different groups. Having been unable to detect the difference in v-rel protein and transcript levels, next we measured the miR-155 level as an indication of v-rel expression, since v-rel is able to induce miR-155 expression as reported previously and in Figure 2b [24,33] (Figure 3d). As expected, miR-155 was upregulated after infection by RCAS(A)-v-rel-GFP and RCAS(A)-v-rel-GFP-NST, whereas the miR-155 level was very low in uninfected cells and RCAS(A)-v-rel-GFP-142T infection when the miR-142 target sequence was present. This indicates that $\mathrm{v}$-rel-induced transformation is inhibited by miRNA targeting. Compared to miR-155 expression, which was significantly upregulated in the transformed cell groups, miR-142 expression levels did not change much during the time course of the transformation (Figure $3 e$ ).

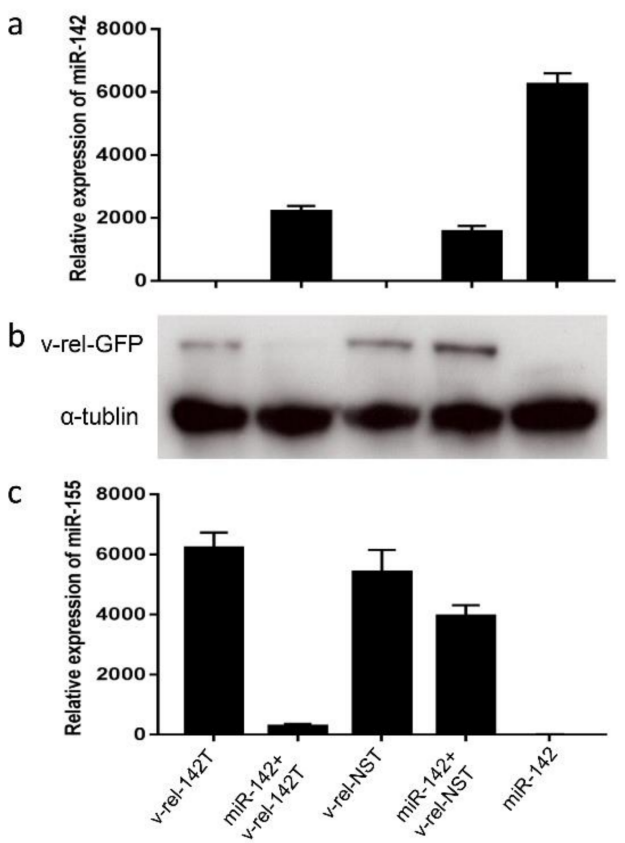

Figure 2. Inhibition of v-rel expression through miR-142 targeting in DF-1. Relative expression of miR-142 (a) and miR-155 (c) measured by qRT-PCR (normalized to let-7a) in RNA extracted from DF-1 infected with RCAS(A)-v-rel-GFP-miRT (142T or NST) and RCAS(B)-GFP-miR-142 singly or in combination. Results represent the mean of triplicate assays with error bars showing the standard errors of the mean. (b) Cell lysates of the same infected DF-1 cells above were analyzed by Western blot using HY87 antibody for v-rel expression. $\alpha$-tubulin was included as a loading control.

miRNA targeting has been shown to be an efficient means to restrict viral replication or attenuate viral pathogenesis in specific tissues, based on different miRNA expression profiles of different cell lineages $[13,19,20,22]$. To our knowledge, this is the first study to demonstrate the effective use of miRNA targeting in inhibiting viral-induced transformation. In this study, we explored the ability of miR-142 to inhibit v-rel-induced transformation by the insertion of miR-142 target sequences downstream of the v-rel gene in RCAS vector. The inhibition effect of v-rel expression was first assessed in DF-1 cells by providing miR-142. Indeed, v-rel-induced transformation in embryonic splenocyte was inhibited. The result shows that the indicator system in DF-1 cells is an effective and useful 
way to assess the inhibition of v-rel-induced oncogenesis. Unpublished data on the inhibition of oncogenesis induced by the oncogene Meq of Marek's disease virus (MDV) confirmed the importance of this strategy, although no in vitro model of the transformation is available. Meq is the major oncoprotein in MDV-induced tumorigenesis. The evolution of viruses towards greater virulence demonstrates the need to introduce newer vaccines in order to keep up with the rapidly evolving viruses. miRNA targeting by incorporating the miR-142 target sequence downstream of Meq would be an attractive way to attenuate $\mathrm{vv}+\mathrm{MDV}$ as a vaccine candidate. Taken together, the results of the present study demonstrate that miRNA targeting can be used to inhibit oncogene v-rel-induced transformation in vitro. This strategy could be applied for the inhibition of oncogenesis induced by other oncogenic viruses, such as Marek's disease virus, to develop molecular defined vaccines.

a

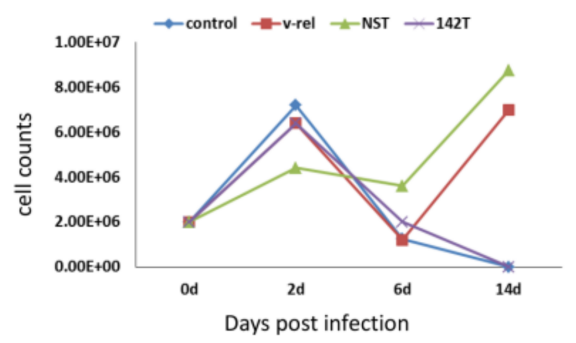

b

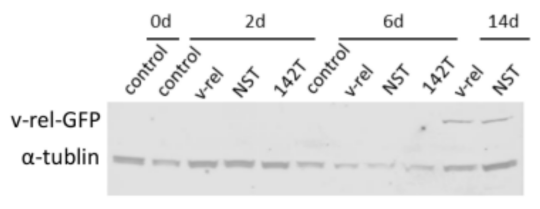

C

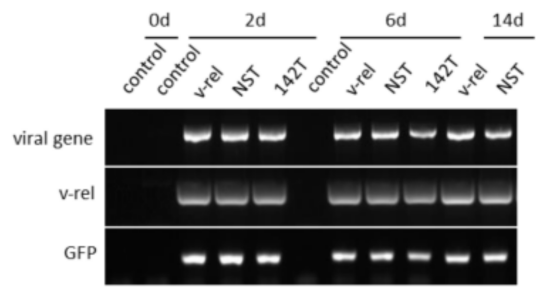

d

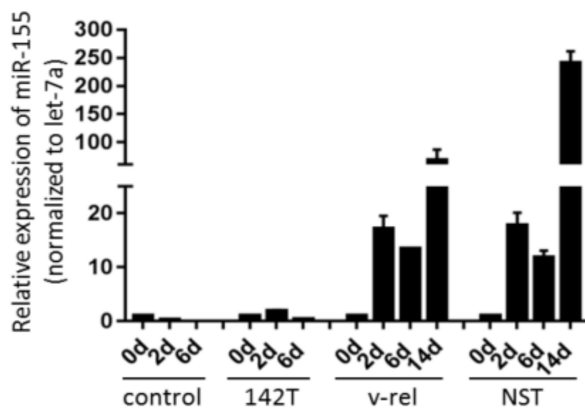

e

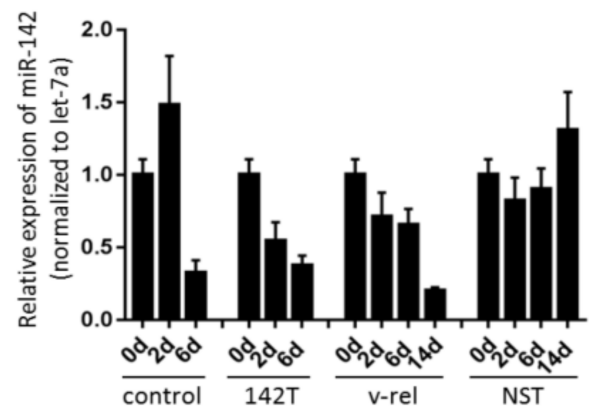

Figure 3. Inhibition of the v-rel-induced transformation of embryonic spenocytes through miR-142 targeting. In brief, $2 \times 10^{6}$ 19-day-old embryonic splenocytes were infected with RCAS(A)-v-rel-GFP-miRT (142T or NST) or RCAS(A)-v-rel-GFP and cells were harvested at days 0, 2, 6, and 14 post-infection for counting, Western blotting, RT-PCR, and miRNA Taqman. (a) Cell counts at different time points during the time course of the transformation; (b) Cell lysates of infected cells harvested at different time points were analyzed by Western blot using HY87 antibody for v-rel expression. $\alpha$-tubulin was included as a loading control; (c) RT-PCR of RNA samples from infected cells using a random primer for reverse transcription and ALV-A-specific primers HA and envA for viral gene detection, v-rel-F and v-rel-R for v-rel detection, and GFP-F and GFP-R for GFP detection; (d,e) Relative expression of miR-155 and miR-142 measured by qRT-PCR (normalized to let-7a) in RNA extracted from infected cells at different time points. Results represent the mean of triplicate assays with error bars showing the standard errors of the mean.

Author Contributions: V.N., Y.Y., and Z.S. conceived and designed experiments; Y.Y., Y.Z., N.T., and M.P. performed experiments; V.N., Y.Y., and Y.Z. analyzed the data; Y.Y. and V.N. wrote the paper.

Acknowledgments: This project was supported by the Biotechnology and Biological Sciences Research Council (BBSRC) grants BB/P016472/1 and BB/K002465/1.

Conflicts of Interest: The authors declare no conflict of interest. 


\section{References}

1. Svoboda, J. Rous sarcoma virus centennial in folia biologica. Folia Biol. 2013, 59, 103-104.

2. Varmus, H. How tumor virology evolved into cancer biology and transformed oncology. Annu. Rev. Cancer Biol. 2017, 1, 1-18. [CrossRef]

3. Nair, V.; Fadly, A. Leukosis/sarcoma group. In Diseases of Poultry, 13th ed.; Swayne, D.E., Glisson, J.R., McDougald, L.R., Nolan, L.K., Suarez, D.L., Nair, V., Eds.; John Wiley \& Sons, Inc.: Ames, IA, USA, 2013; pp. 553-592.

4. Pishkari, S.; Paryan, M.; Hashemi, M.; Baldini, E.; Mohammadi-Yeganeh, S. The role of microRNAs in different types of thyroid carcinoma: A comprehensive analysis to find new miRNA supplementary therapies. J. Endocrinol. Investig. 2018, 41, 269-283. [CrossRef] [PubMed]

5. Bartel, D.P. MicroRNAs: Target recognition and regulatory functions. Cell 2009, 136, 215-233. [CrossRef] [PubMed]

6. Filipowicz, W.; Bhattacharyya, S.N.; Sonenberg, N. Mechanisms of post-transcriptional regulation by microRNAs: Are the answers in sight? Nat. Rev. Genet. 2008, 9, 102-114. [CrossRef] [PubMed]

7. Lagos-Quintana, M.; Rauhut, R.; Yalcin, A.; Meyer, J.; Lendeckel, W.; Tuschl, T. Identification of tissue-specific microRNAs from mouse. Curr. Biol. 2002, 12, 735-739. [CrossRef]

8. Ebert, M.S.; Sharp, P.A. MicroRNA sponges: Progress and possibilities. RNA 2010, 16, 2043-2050. [CrossRef] [PubMed]

9. Fang, Y.; Zhou, Y.; Zhang, Y.; He, L.; Xue, C.; Cao, Y. Design of miRNA sponges for MDV-1 as a therapeutic strategy against lymphomas. Oncotarget 2018, 9, 3842-3852. [CrossRef] [PubMed]

10. Gentner, B.; Schira, G.; Giustacchini, A.; Amendola, M.; Brown, B.D.; Ponzoni, M.; Naldini, L. Stable knockdown of microRNA in vivo by lentiviral vectors. Nat. Methods 2009, 6, 63-66. [CrossRef] [PubMed]

11. Tay, F.C.; Lim, J.K.; Zhu, H.; Hin, L.C.; Wang, S. Using artificial microRNA sponges to achieve microRNA loss-of-function in cancer cells. Adv. Drug Deliv. Rev. 2015, 81, 117-127. [CrossRef] [PubMed]

12. Brown, B.D.; Cantore, A.; Annoni, A.; Sergi, L.S.; Lombardo, A.; Della Valle, P.; D'Angelo, A.; Naldini, L. A microRNA-regulated lentiviral vector mediates stable correction of hemophilia B mice. Blood 2007, 110, 4144-4152. [CrossRef] [PubMed]

13. Edge, R.E.; Falls, T.J.; Brown, C.W.; Lichty, B.D.; Atkins, H.; Bell, J.C. A let-7 microRNA-sensitive vesicular stomatitis virus demonstrates tumor-specific replication. Mol. Ther. 2008, 16, 1437-1443. [CrossRef] [PubMed]

14. Kelly, E.J.; Hadac, E.M.; Greiner, S.; Russell, S.J. Engineering microRNA responsiveness to decrease virus pathogenicity. Nat. Med. 2008, 14, 1278-1283. [CrossRef] [PubMed]

15. Kelly, E.J.; Russell, S.J. MicroRNAs and the regulation of vector tropism. Mol. Ther. 2009, 17, 409-416. [CrossRef] [PubMed]

16. TenOever, B.R. RNA viruses and the host microRNA machinery. Nat. Rev. Microbiol. 2013, 11, 169-180. [CrossRef] [PubMed]

17. Brown, B.D.; Venneri, M.A.; Zingale, A.; Sergi Sergi, L.; Naldini, L. Endogenous microRNA regulation suppresses transgene expression in hematopoietic lineages and enables stable gene transfer. Nat. Med. 2006, 12, 585-591. [CrossRef] [PubMed]

18. Barnes, D.; Kunitomi, M.; Vignuzzi, M.; Saksela, K.; Andino, R. Harnessing endogenous miRNAs to control virus tissue tropism as a strategy for developing attenuated virus vaccines. Cell Host Microbe 2008, 4, $239-248$. [CrossRef] [PubMed]

19. Cawood, R.; Wong, S.L.; Di, Y.; Baban, D.F.; Seymour, L.W. MicroRNA controlled adenovirus mediates anti-cancer efficacy without affecting endogenous microRNA activity. PLoS ONE 2011, 6, e16152. [CrossRef] [PubMed]

20. Kelly, E.J.; Hadac, E.M.; Cullen, B.R.; Russell, S.J. MicroRNA antagonism of the picornaviral life cycle: Alternative mechanisms of interference. PLoS Pathog. 2010, 6, e1000820. [CrossRef] [PubMed]

21. Perez, J.T.; Pham, A.M.; Lorini, M.H.; Chua, M.A.; Steel, J.; tenOever, B.R. MicroRNA-mediated species-specific attenuation of influenza a virus. Nat. Biotechnol. 2009, 27, 572-576. [CrossRef] [PubMed]

22. Teterina, N.L.; Maximova, O.A.; Kenney, H.; Liu, G.; Pletnev, A.G. MicroRNA-based control of tick-borne flavivirus neuropathogenesis: Challenges and perspectives. Antivir. Res. 2016, 127, 57-67. [CrossRef] [PubMed]

23. Hughes, S.H. The rcas vector system. Folia Biol. 2004, 50, 107-119. 
24. Yao, Y.; Vasoya, D.; Kgosana, L.; Smith, L.P.; Gao, Y.; Wang, X.; Watson, M.; Nair, V. Activation of gga-miR-155 by reticuloendotheliosis virus $\mathrm{T}$ strain and its contribution to transformation. J. Gen. Virol. 2017, 98, 810-820. [CrossRef] [PubMed]

25. Chen, C.Z.; Li, L.; Lodish, H.F.; Bartel, D.P. MicroRNAs modulate hematopoietic lineage differentiation. Science 2004, 303, 83-86. [CrossRef] [PubMed]

26. Shrestha, A.; Carraro, G.; El Agha, E.; Mukhametshina, R.; Chao, C.M.; Rizvanov, A.; Barreto, G.; Bellusci, S. Generation and validation of miR-142 knock out mice. PLoS ONE 2015, 10, e0136913. [CrossRef] [PubMed]

27. Lambeth, L.S.; Yao, Y.; Smith, L.P.; Zhao, Y.; Nair, V. MicroRNAs 221 and 222 target p27Kip1 in marek's disease virus-transformed tumour cell line MSB-1. J. Gen. Virol. 2009, 90, 1164-1171. [CrossRef] [PubMed]

28. Gilmore, T.D.; Wolenski, F.S. NF-кB: Where did it come from and why? Immunol. Rev. 2012, 246, 14-35. [CrossRef] [PubMed]

29. Liss, A.S.; Tiwari, R.; Kralova, J.; Bose, H.R., Jr. Cell transformation by v-rel reveals distinct roles of AP-1 family members in Rel/NF-kB oncogenesis. Oncogene 2010, 29, 4925-4937. [CrossRef] [PubMed]

30. Zhang, Q.; Lenardo, M.J.; Baltimore, D. 30 years of NF-kB: A blossoming of relevance to human pathobiology. Cell 2017, 168, 37-57. [CrossRef] [PubMed]

31. Hrdlickova, R.; Nehyba, J.; Humphries, E.H. V-rel induces expression of three avian immunoregulatory surface receptors more efficiently than c-rel. J. Virol. 1994, 68, 308-319. [PubMed]

32. Fenton, S.P.; Reddy, M.R.; Bagust, T.J. Single and concurrent avian leukosis virus infections with avian leukosis virus-J and avian leukosis virus-A in Australian meat-type chickens. Avian Pathol. 2005, 34, 48-54. [CrossRef] [PubMed]

33. Bolisetty, M.T.; Dy, G.; Tam, W.; Beemon, K.L. Reticuloendotheliosis virus strain T induces miR-155, which targets JARID2 and promotes cell survival. J. Virol. 2009, 83, 12009-12017. [CrossRef] [PubMed]

(C) 2018 by the authors. Licensee MDPI, Basel, Switzerland. This article is an open access article distributed under the terms and conditions of the Creative Commons Attribution (CC BY) license (http:/ / creativecommons.org/licenses/by/4.0/). 